\title{
Observation of the fractional quantum Hall effect in $\mathrm{GaAs}-(\mathrm{Ga}, \mathrm{Al}) \mathrm{As}$ quantum well structures
}

\author{
C.V. Brown, C.J.G.M. Langerak, P.C. Main, L. Eaves, T.J. Foster and M. Henini \\ Department of Physics, University of Nottingham, UK
}

\author{
P.A.A. Teunissen and J.A.A.J. Perenboom \\ High Field Magnet Laboratory and Research Institute for Materials, University of Nijmegen, The Netherlands
}

\begin{abstract}
We report the observation of the fractional quantum Hall effect in single $\mathrm{GaAs}-(\mathrm{Ga}, \mathrm{Al})$ As quantum well structures with well widths of $102 \AA$ and $68 \AA$. In both samples we have observed a strong $\nu=4 / 3$ fraction in both the longitudinal resistivity $\rho_{x x}$ and the Hall resistivity $\rho_{x y}$. The surprising result in our data (in comparison with the FQHE in conventional single heterojunctions) is that the $5 / 3$ is heavily suppressed or possibly absent. In a tilted magnetic field the energy gap of $4 / 3$ depends only on the perpendicular field. We associate this difference between our samples and the conventional heterojunctions with the energy (well width) dependence of the Lande $g^{*}$ factor and the influence of the Zeeman energy on the (partially) spin-polarized energy levels and hence the activation energies.
\end{abstract}

Recent experiments on the fractional quantum Hall effect (FQHE) have focussed on the determination of the spin configuration of the electron ground state $[1,2]$ and the energy gap $\Delta$ $[3,4]$ above this ground state. Initially it was thought that the ground state of the FQHE was spin-polarised, but recent theoretical [5] and experimental $[1,2]$ investigations in tilted magnetic fields have shown that spin-unpolarised states are also possible. It was found that the $\frac{5}{3}$ fraction has a spin-polarised ground state, whilst the $\frac{4}{3}$ fraction has a spin-unpolarised ground state [1]. In conventional heterojunctions used to study the FQHE, the spatial extent of the electron wavefunction is large $(\sim 250 \AA)$. A (Ga,Al)AsGaAs-(Ga,Al)As quantum well makes it possible to compress further the wavefunction. However, the scattering on the second interface tends to suppress the FQHE. Nevertheless, there have been some investigations of the FQHE in $\mathrm{GaAs}-(\mathrm{Ga}, \mathrm{Al}) \mathrm{As}$ multi quantum well systems

Correspondence to: P.C. Main, Department of Physics, University of Nottingham, University Park, Nottingham NG7 2RD, UK.
[6] and wide single quantum well systems $[4,7]$.

In this paper we study the FQHE in a $2 \mathrm{DEG}$ confined in a narrow single quantum well. The filling factors $\nu=\frac{4}{3}$ and $\frac{5}{3}$ show a large difference in their activation energies. A similar effect has been found in hydrostatic pressure experiments on conventional high-mobility single heterostructures by Morawicz et al. [8,9]. Their data show a well-developed $\frac{5}{3}$ minimum, which is unchanged with pressure. The $\frac{4}{3}$ state, however, is not present at atmospheric pressure, but develops into a very strong feature by applying pressures up to $9 \mathrm{kbar}$. Because of the energy dependence of the Landé $g^{*}$ factor one can change the value of $g^{*}$ by changing the pressure $[8,9]$ or the well width, as optical experiments on these type of samples have shown [10]. We consider the reduction of the Zeeman energy as a possible explanation of the striking difference between our data and those for conventional heterojunctions.

The samples are MBE-grown GaAs(Ga, Al)As single quantum wells, modulation doped on one side of the well [7], with well widths of $L_{\mathrm{w}}=102 \AA$ and $68 \AA$. The carrier con- 
centrations and mobilities $\left(N_{\mathrm{s}}=1-3.4 \times\right.$ $10^{15} \mathrm{~m}^{-2}$ and $\mu=37-55 \mathrm{~m}^{2} / \mathrm{Vs}$ for the $102 \AA$ well and $N_{\mathrm{s}}=1.8-3.1 \times 10^{15} \mathrm{~m}^{-2}$ and $\mu=36-$ $52 \mathrm{~m}^{2} / \mathrm{V} \mathrm{s}$ for the $68 \AA$ well) could be varied by illumination with an infrared (sub-bandgap) LED.

Figure 1 shows the diagonal resistance $R_{x x}$ and Hall resistance $R_{x y}$ of the $68 \AA$ sample as a function of the magnetic field. The $\frac{4}{3}$ fraction can (over the whole electron density range and in both samples) unambiguously be identified by the value of the corresponding Hall plateau. A slightly puzzling feature in these samples is that the magnetic field position of the $\frac{4}{3}$ minimum differs slightly $(\sim 5 \%)$ from the extrapolation of the low field Shubnikov-De Haas oscillations. The $\frac{5}{3}$ fraction is found in the same way in the $102 \AA$ well. In the $68 \AA$ well, however, the features in $R_{x x}$ and $R_{x y}$ at magnetic fields just below the $\frac{4}{3}$ fraction are slightly off from what is expected for the $\frac{5}{3}$ fraction in the field value as well as in the Hall resistance value. An identification of

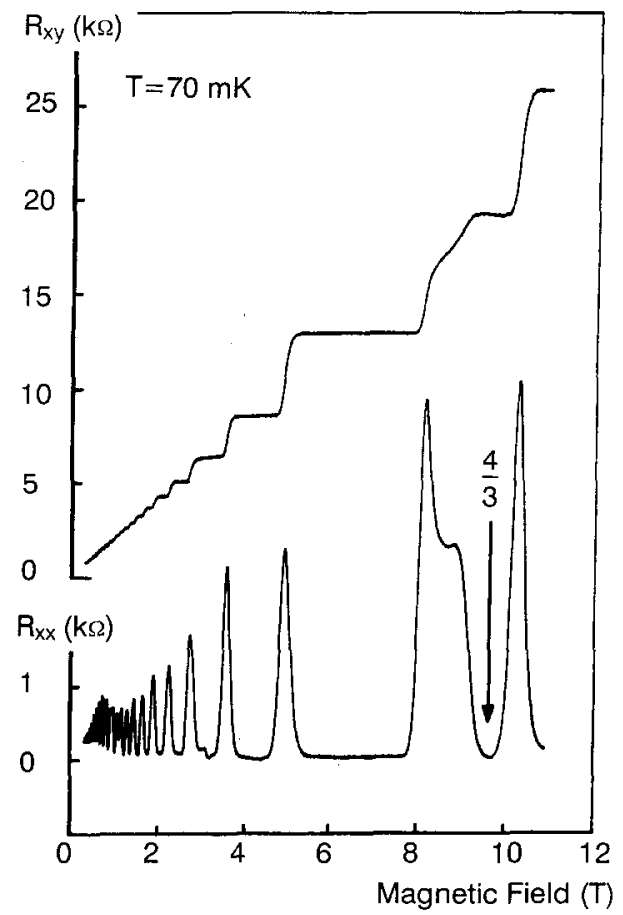

Fig. 1. Diagonal and Hall resistances $R_{x x}$ and $R_{x y}$ for the $68 \AA$ well width sample. The density is $N_{\mathrm{s}}=3.2 \times 10^{15} \mathrm{~m}^{-2}$ and the mobility is $\mu=40 \mathrm{~m}^{2} / \mathrm{Vs}$. $\frac{3}{2}$ could also be possible; in that case the $\frac{5}{3}$ is completely absent in this sample. In this paper we focus on the $\frac{4}{3}$ and $\frac{5}{3}$ and the remarkable difference in their relative strength. In conventional single heterojunctions these two fractions appear with a similar strength, while in this experiment the $\frac{4}{3}$ fraction is much better developed than the $\frac{5}{3}$ fraction (which might even be absent in the case of the $68 \AA$ well). The activation energy $\Delta$ is determined from temperature dependent measurements assuming $\rho_{x x}(T)=$ $\rho_{x x}^{\mathrm{c}} \mathrm{e}^{-\Delta / k T}$. Figure 2 shows measurements for several temperatures on the $68 \AA$ sample at two different electron densities. The $\frac{4}{3}$ fraction when it occurs at $9.0 \mathrm{~T}$ has an activation energy $\Delta=$ $0.2 \mathrm{~K}$ (fig. 2(a)), at least an order of magnitude larger than the activation energy of the feature at slightly higher filling factor when it occurs at a similar field (fig. 2(b)). A direct comparison between samples is very difficult because the activation energies are masked by disorder, Landau-level mixing and the finite width of the 2DEG, but similar effects have also been observed in the $102 \AA$ well sample.

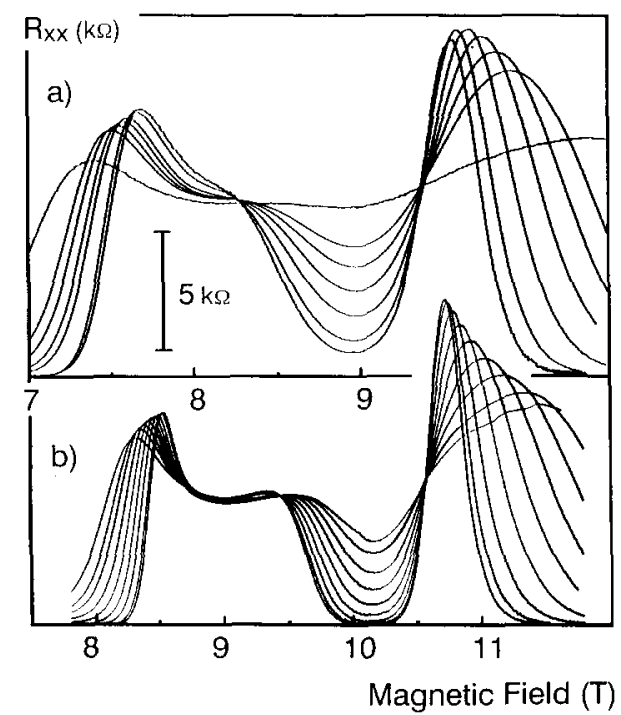

Fig. 2. Temperature dependence of $R_{x x}$ around $\nu=\frac{4}{3}$ at two different densities of the $68 \AA$ sample: (a) $N_{\mathrm{s}}=2.8 \times 10^{15} \mathrm{~m}^{-2}$ (the temperatures are $75,113,169,243,307,390$ and $820 \mathrm{mK}$ respectively) and (b) $N_{\mathrm{s}}=3.1 \times 10^{15} \mathrm{~m}^{-2}$ (the temperatures are $66,91,124,161,214,281,349,433$ and $518 \mathrm{mK}$ respectively). 
For an ideal (infinitely thin) $2 \mathrm{DEG}$ the field dependence of the activation energy is given by $\Delta=0.1 e^{2} / \varepsilon l_{0}$ (for filling factors $\frac{1}{3}, \frac{2}{3}, \frac{4}{3}$ and $\frac{5}{3}$ ) where $\varepsilon$ is the dielectric constant and $l_{0}=$ $(\hbar / e B)^{1 / 2}$ is the magnetic length [11]. However, this theory does not take into account the possibility of partially polarised and unpolarised ground states and excited states with polarisation different from the ground state. The total energy of the interacting, ideal 2DEG consists of a Coulomb term and a Zeeman term [5], namely

$E=E_{\mathrm{C}}(S, B \cos \theta)+g^{*} \mu_{\mathrm{B}} B S$

where $S$ is the spin quantum number, $g^{*}$ is the effective bare Landé $g^{*}$ factor. All the manyparticle effects, leading for example to exchange enhancement, are included in the Coulomb term $E_{\mathrm{C}}$ (which has the $\sqrt{B}$ dependence for an ideal 2DEG). The spin state of a 2D system with $N_{\mathrm{s}}$ electrons can vary between its minimum value 0 (unpolarised) and its maximum $\frac{1}{2} N_{\mathrm{s}}$ (polarised) in integer steps $\Delta S=1$. Finite-size calculations of $E_{\mathrm{C}}$ by Maksym [5] show that it is possible to have partially polarised and unpolarised ground states and that the first excited state can have a polarisation different from the ground state. The Zeeman energy can have a considerable contribution to the activation energy $\Delta$ if the ground state and first excited state have a different polarisation. In general we can write

$\Delta=\left(E_{1}-E_{0}\right) \pm \Delta S g^{*} \mu_{\mathrm{B}} B$

where $E_{0}$ and $E_{1}$ are the Coulomb terms of eq. (1) of the ground and first excited states of the FQHE spectrum (which can have different polarisations) and $\Delta S$ is the change in spin state of the system in going from the ground state to the first excited state. $E_{1}-E_{0}$ has the $\sqrt{B}$ dependence for an ideal $2 \mathrm{DEG}$ as discussed above. The \pm -sign indicates an increase or decrease in polarisation in going from the ground state to the first excited state.

The Lande $g^{*}$ factor in eqs. (1) and (2) is small and negative in bulk GaAs $\left(g^{*}=-0.44\right)$. From $k \cdot p$ perturbation theory [12], it has an energy dependence given by

$\frac{g^{*}}{g}-1=-\frac{P^{2}}{3}\left(\frac{1}{E}-\frac{1}{E-\Delta}\right)+$ h.o.t.

where $g_{0}=2$ is the free-electron Landé factor, $E_{g}$ is the bandgap energy, $\Delta_{0}$ is the valence-band spin-orbit splitting and $P^{2}$ describes the coupling between the conduction band and the other bands. In a simple approximation for a quantum well system, we can add the subband energy $E_{\text {sub }}$ to the bandgap energy $E_{\mathrm{g}}$. We find that $\left|g^{*}\right|$ is then reduced in magnitude for a smaller well width and becomes zero at a well width of $L_{\mathrm{w}}=22 \AA$. This decrease in $\left|g^{*}\right|$ has been measured by photoluminesence [10]. Although the values do not fit eq. (4) exactly, the data show similar qualitative behaviour: the value of $g^{*}$ increases with increasing confinement and changes sign for quantum wells with $L_{\mathrm{w}} \approx 55 \AA$. The commonly used heterojunctions have a 'width' of the $2 \mathrm{DEG}$ which is of order $250 \AA$. Therefore, the value of the effective $\left|g^{*}\right|$ factor, and thus the Zeeman energy $g^{*} \mu_{\mathrm{B}} B$, will be smaller in our samples.

The detailed implications of this on our experiment depends on the polarisation of the ground state. For a heterostructure the $\frac{4}{3}$ ground state is spin-unpolarized at low magnetic fields, but changes into a polarised ground state at higher fields, whereas the $\frac{5}{3}$ ground state is spin-polarised over the whole field range $[1,5,13]$. An unpolarised state cannot go to a lower spin configuration and a fully polarised state cannot go to a higher spin configuration. The consequence of this on the activation energy is shown schematically in fig. 3. Here the energy levels for a polarised $\left(S=\frac{1}{2} N_{\mathrm{s}}\right)$ and an unpolarised $(S=0)$ ground state are drawn. In each case there are two possibilities for activated transport: there is no spin flip involved in going from the ground state to the first excited state $(\Delta S=0)$ or the first excited state has a different polarisation and $\Delta S \neq 0$. The Coulomb term $E_{\mathrm{C}}$ of eq. (1) is represented by the solid levels. The Zeeman term will reduce these energy levels by $g^{*} \mu_{\mathrm{B}} B S$, with a value depending on the degree of polarisation $S$, resulting in the dashed lines. A reduc- 


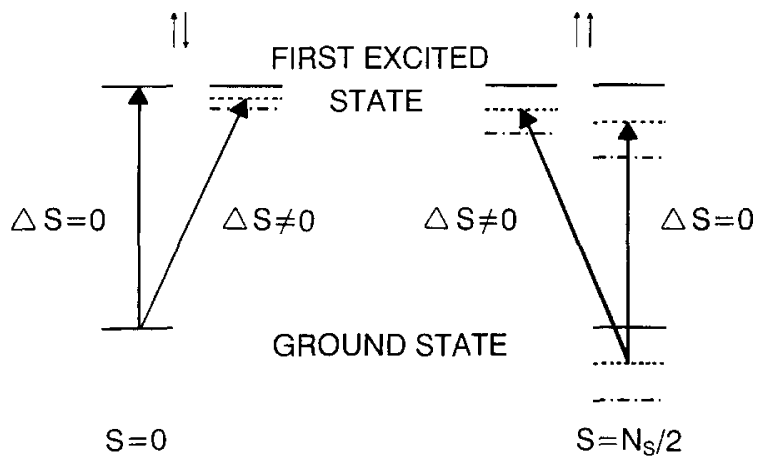

Fig. 3. Schematic diagram of the FQHE energy levels: for the case when the absolute ground state is unpolarised $(S=0)$ and completely polarised $\left(S=\frac{1}{2} N_{\mathrm{s}}\right)$. The solid lines represent the Coulomb term $E_{\mathrm{C}}$ from eq. (2); adding the Zeeman energy $g^{*} \mu_{\mathrm{B}} B S$ to $E_{\mathrm{C}}$ gives the dashed lines, and the dotted lines are the levels when $\left|g^{*}\right|$ is reduced. The arrows represent possible activation processes corresponding to changes of total spin $\Delta S$.

tion of $\left|g^{*}\right|$ will reduce the Zeeman term, and consequently enhance the energy levels indicated by the dotted lines, resulting (if $\Delta S \neq 0$ ) in a decrease of $\Delta$ if the groundstate is polarised and an increase of $\Delta$ if the groundstate is unpolarised.

Since the $\frac{5}{3}$ fraction has a polarised ground state we expect a lower activation energy in narrow quantum wells than in heterojunctions if $\Delta S \neq 0$, consistent with our data. In conventional heterojunctions the $\frac{4}{3}$ can have an unpolarised and polarised groundstate, depending on, for example, the value of the magnetic field. It is not clear what the polarisation of the $\frac{4}{3}$ in our experiment is. Figure 4 shows the combined magnetic field/density dependence of the activation energy of the $\frac{4}{3}$ fraction of the $68 \AA$ well sample. Also data for magnetic fields tilted $\sim 30^{\circ}$ and $\sim 35^{\circ}$ from the normal of the $2 \mathrm{DEG}$ are shown. The closed symbols represent the activation energies as function of total magnetic field; the open symbols refer to the same data but now the horizontal axis is the perpendicular component of the magnetic field. The value of the activation energy increases with $B$ but one must realise that changing the electron density not only affects the Zeeman energy in eq. (1), but also the Coulomb energy $E_{\mathrm{C}}$ (which contains many-particle effects).
In ref. [1] it is mentioned that in their particular case the effect of changing the electron density is similar to the effect of changing the tilt angle of the sample with respect to the magnetic field. This is not the case in our sample, where the activation energy only depends on the perpendicular component of the magnetic field and not the total field (fig. 4). This implies that the Zeeman contribution to the activation energy in eq. (2) is negligible. Thus $g^{*}=0$ or $\Delta S=0$ in our samples. Snelling et al. [10] have measured the $g^{*}$ factor in quantum wells, and from their data one finds $g^{*} \approx-0.1$ for a $70 \AA$ well. It is not quite clear from our data what the polarisation of the $\frac{4}{3}$ ground state is. We could be in the range where changing the electron density decreases the disorder significantly, leading to an increase in the activation energy.

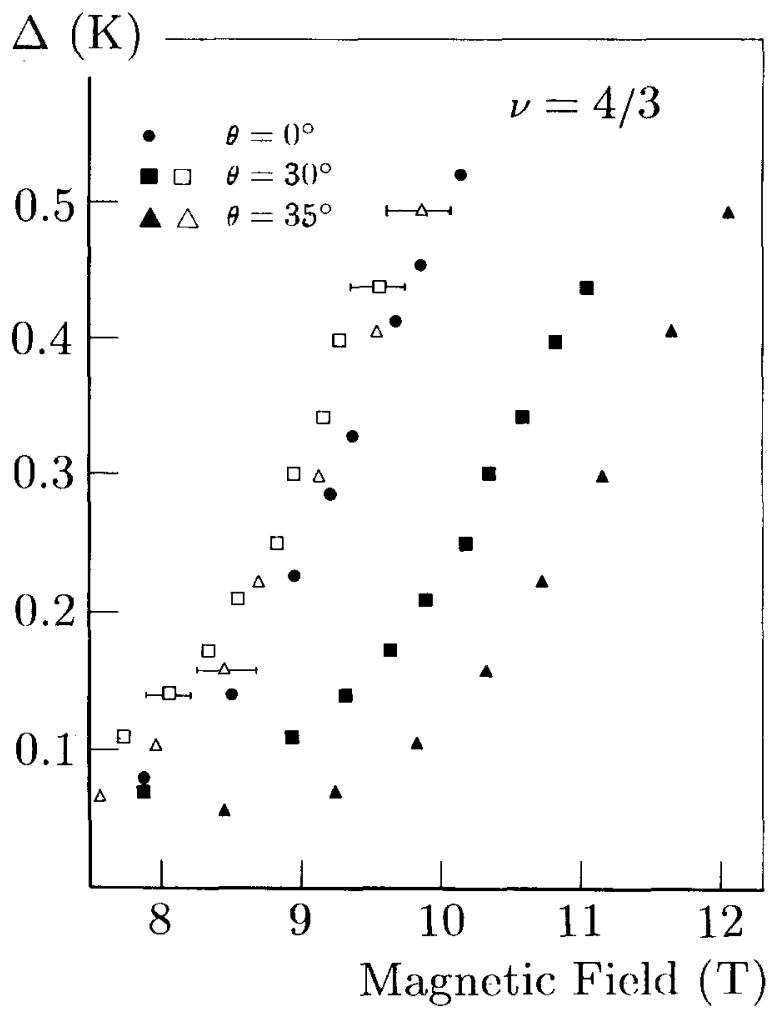

Fig. 4. Magnetic field dependence of the activation energy of the $\frac{4}{3}$ fraction for the sample with well width $L_{\mathrm{w}}=68 \AA$ at three different angles. For the filled symbols the magneticfield axis refers to the total field and for the open symbols to the perpendicular component. 
Instead of changing the well width, one can also apply pressure to reduce $g^{*}$. Similar effects have been found in hydrostatic pressure experiments on conventional single heterojunctions by Morawicz et al. [8,9]. This results in a better developed $\frac{4}{3}$ fraction at higher pressures. The idea of spin-flip activation energies was already suggested in the tilted-field experiments of Eisenstein [2] and Furneaux [14]. Here we have added the well width as a parameter to vary the value of $g^{*}$.

Another remarkable feature is visible in fig. 2: at certain values of magnetic field just above and below $\nu=\frac{4}{3}$ there is no temperature dependence of the diagonal resistance $R_{x x}$. All the different temperature curves cross at the same point. Such single crossing points are observed for the whole range of electron densities; two examples are shown in fig. 2. It is clear that this feature is related to the FQHE, since it does not appear near the integer filling factors. The temperature independent resistance appears to be due to an exact compensation between two competing thermally activated processes, such as hopping through the $2 \mathrm{D}$ bulk states and activated transport across the FQH energy gap.

To summarize, our experiment and the highpressure experiment $[8,9]$ suggest that one must take the polarisation and Zeeman energy (well width dependence of $g^{*}$ ) of ground and excited states into account. Because of the low value for the Lande $g^{*}$ factor, these samples allow the possibility of studying the many-particle effects in the FQHE, without the Zeeman energy being the dominant term.
We would like to thank Dr. P. Maksym, Dr. R. Harley and Dr. K. Barnham for helpful discussions. This work was funded by the SERC (UK) and FOM (the Netherlands).

\section{References}

[1] R.G. Clark, S.R. Haynes, A.M. Suckling, J.R. Mallet, P.A. Wright, J.J. Harris and C.T. Foxon, Phys. Rev. Lett. 62 (1989) 1536.

[2] J.P. Eisenstein, H.L. Stormer, L. Pfeiffer and K.W. West, Phys. Rev. Lett. 62 (1989) 1540.

[3] R.L. Willet, H.L. Stormer, D.C. Tsui, A.C. Gossard and J.H. English, Phys. Rev. B 37 (1988) 8476.

[4] M. Shayegan, J. Jo, W. Suen, M. Santos and V.J. Goldman, Phys. Rev. Lett. 65 (1990) 2916.

[5] P.A. Maksym, J. Phys.: Condens. Matter 1 (1989) 6299.

[6] B.B. Goldberg, D. Heiman, A. Pinczuk, C.W. Tu, A.C. Gossard and J.H. English, Surf. Sci. 196 (1988) 209.

[7] C.R.H. White, M. Davies, M. Henini, B.R. Davidson, P.C. Main, J.R. Owers-Bradley, L. Eaves, O.H. Hughes, M. Heath and M.S. Skolnick, Semicond. Sci. Technol. 5 (1990) 792.

[8] N.G. Morawicz, K.W.J. Barnham, C. Zammit, J.J. Harris, C.T. Foxon and P. Kujawinski, Phys. Rev. B 41 (1990) 12687.

[9] N.G. Morawicz, K.W.G. Barnham, A. Briggs, S. Najda, J.C. Portal, J.J. Harris and C.T. Foxon, preprint.

[10] M.J. Snelling, G.P. Flinn, A.S. Plaut, R.T. Harley, A.C. Tropper, R. Eccleston and C.C. Phillips, Phys. Rev. B 44 (1991) 11345.

[11] See, e.g., F.D.M. Haldane, in: The Quantum Hall Effect, eds. R.E. Prange and S.M. Girvin (SpringerVerlag, Berlin, 1987).

[12] C. Hermann and C. Weisbuch, Phys. Rev. B 15 (1977) 823.

[13] R.G. Clark, Phys. Scripta T 39 (1991) 45.

[14] J.E. Furneaux, D.A. Syphers and A.G. Swanson, Phys. Rev. Lett. 63 (1989) 1098. 